\title{
Tissue Doppler Study before and after PCI in Patients with Chronic Stable Angina and Apparent Normal Ventricular Function for Evaluation of Myocardial Function
}

\author{
Hossam Eldin M. Mahmoud1, Ahmed Boghdady2, Mohamed A. Alsenbesy¹, Alaa A. Ghalib ${ }^{3}$, \\ Ahmed Okasha ${ }^{4}$, Huda A. Dardeer ${ }^{1}$, Ahlam M. Sabra ${ }^{1}$ \\ ${ }^{1}$ Internal Medicine Department, Qena Faculty of Medicine, South Valley University, Qena, Egypt \\ ${ }^{2}$ Cardiology Department, Sohag Faculty of Medicine, Sohag University, Sohag, Egypt \\ ${ }^{3}$ Internal Medicine Department, Sohag Faculty of Medicine, Sohag University, Sohag, Egypt \\ ${ }^{4}$ Radiodiagnosis Department, Qena Faculty of Medicine, South Valley University, Qena, Egypt \\ Email: dr_hossam_ismail@yahoo.com
}

How to cite this paper: Mahmoud, H.E.M., Boghdady, A., Alsenbesy, M.A., Ghalib, A.A., Okasha, A., Dardeer, H.A. and Sabra, A.M. (2020) Tissue Doppler Study before and after PCI in Patients with Chronic Stable Angina and Apparent Normal Ventricular Function for Evaluation of Myocardial Function. World Journal of Cardiovascular Diseases, 10, 395-409. https://doi.org/10.4236/wjcd.2020.107038

Received: May 18, 2020

Accepted: July 11, 2020

Published: July 14, 2020

Copyright $\odot 2020$ by author(s) and Scientific Research Publishing Inc. This work is licensed under the Creative Commons Attribution International License (CC BY 4.0).

http://creativecommons.org/licenses/by/4.0/

(c) (i) Open Access

\begin{abstract}
Background: This study aimed to determine the impact of the percutaneous coronary intervention (PCI) on myocardial function assessed by tissue Doppler echocardiography. Methods: Conventional two-dimensional echocardiography and Myocardial tissue peak velocities were recorded at the lateral, ant.septal, post.septal, posterior, ant. and inferior angles of the mitral annulus as well as at the lateral tricuspid annulus by pulsed-wave tissue Doppler echocardiography before PCI, as well as 1 day and 6 weeks after intervention. Results: Fifty consecutive patients with chronic stable angina and preserved systolic left ventricular function (mean age, $58.3 \pm 6.594$ years; 32 men) undergoing PCI were studied. Conventional echocardiographic revealed no statistically significant difference between pre- and post-PCI (1 day after PCI and 6 weeks after PCI) as regarding trans-mitral and trans-tricuspid flow velocities except as regarding LVEDD, LVESD and LVEF which showed a significant improvement post-PCI. Compared with pre-interventional values, systolic peak velocity and early diastolic velocities improved at all sites $(\mathrm{P} \leq$ 0.05 for each). The most pronounced improvement occurred in the septal area. Similarly, late diastolic velocities improved at all sites ( $\mathrm{P} \leq 0.05$ for each) except at post Wall A'. Also, there was a significant improvement of E'DT \& $E^{\prime} / A^{\prime}$ ratio after PCI ( $\mathrm{P}$ value is significant $<0.05$ for each) but there was insignificant difference between pre \& post PCI for E/E' $(\mathrm{P}$-value $=0.154)$. There was a significant improvement in tissue Doppler measures of early, late diastolic function and E'/A' at the lateral tricuspid annulus after PCI com-
\end{abstract}


pared with baseline values $(\mathrm{P}$ value is significant $<0.05)$ but there was an insignificant change in tissue Doppler measures at the lateral tricuspid annulus of E'DT \& E/E' after PCI (repeated measures ANOVA P-value is $>0.05$ for each). There were significant reductions in IVRT, IVCT \& MPI after PCI (P value is significant $<0.05$ for each) but there was insignificant change in ET after PCI $(\mathrm{P}$-value $=0.09)$ at the septal angle of the mitral annulus and the lateral angle of the tricuspid annulus. Conclusions: Tissue Doppler parameters of diastolic and systolic function improve early after successful PCI, and this effect persists to 6 weeks after the intervention that emphasizes its value in the improvement of regional and global LV functions and myocardial contractility.

\section{Keywords}

Doppler Tissue Imaging, Coronary Artery Disease, Percutaneous Coronary Intervention

\section{Introduction}

Percutaneous coronary intervention (PCI) is now an established and widely agreed technique for the treatment of coronary artery disease (CAD); with worthy role in symptom relief. Yet, the effect of PCI on cardiac fubctions (either systolic or diastolic) among patients with conserved baseline left ventricular systolic function is largely still unknown [1].

Tissue Doppler Imaging (TDI) echocardiography can be used as a sensitive tool for both systolic and diastolic longitudinal myocardial function; providing also quantitative measures for these functions \& has proved to be a useful prognostic tool both in the general population and among persons with known cardiovascular disease [2] [3].

Additionally, impaired left ventricular longitudinal function may precede circumferential ventricular dysfunction in patients with CAD [4]. Evaluation of myocardial contractile function (either global or regional) using TDI echocardiography chiefly depends on visual evaluation of the extent of myocardial thickening and endocardial inward motion, along with both M-mode and two-dimensional echocardiographic measurements of left ventricular (LV) ejection fraction (LVEF) [5].

Although TDI echocardiography has been used for many decades; its clinical application is still controversial. Unlike conventional visual assessment of regional wall motion abnormalities, requiring subjective interpretation, tissue Doppler velocities can be quantified objectively. The potential of tissue Doppler-derived measurements in identifying ischemia has been established in different experimental and clinical settings so, PCI for a significant coronary lesion with chronic stable angina has a beneficial effect on LV functions and improvement in regional and global LV functions and myocardial contractility after re- 
vascularization, which can be predicted by conventional echo and more accurate and more details can be obtained by tissue Doppler study (TDI) [6].

\section{Aim of the work.}

This study aimed to estimate the impact of the PCI on myocardial function assessed by TDI echocardiography in patients with chronic stable angina.

\section{Methods}

\section{Study Population}

Fifty (50) consecutive patients with chronic stable angina and preserved left ventricular systolic function (EF\% more than 50\%) and underwent for PCI at south valley university hospitals, faculty of medicine, south valley university, were included in this prospective study and were studied by pulsed wave tissue doppler during the period from September 2018 to January 2020.

This study was approved by the local ethics committee, and all patients provided informed consent. All patients had regional wall motion index scores of 1 and normal left ventricular systolic function and end-diastolic and end-systolic dimensions. Patients who had LV systolic dysfunction by echocardiography (EF\% less than 50\%) and patients who had a prior myocardial infarction or acute coronary syndrome were excluded from this study.

\section{Coronary Angiography and PCI}

Patients underwent coronary angiography and PCI in a single session at Qena University Hospitals. The performed intervention, as well as the choice whether or not to use coronary stents, was at the treating cardiologists' decision.

\section{Echocardiography}

Echocardiographic images were obtained using GE Vivid S5 with a $3.5-\mathrm{MHz}$ transducer. All subjects were examined with conventional two-dimensional echocardiography and pulsed waved TDI according to a standardized study protocol.

\section{1) Conventional echocardiography}

Conventional echocardiography was performed before PCI (24 hours before the procedure) as well immediately after the procedure (1 day after the procedure in all subjects). An additional conventional echocardiographic examination was performed 6 weeks after PCI. Left ventricular (LV) diameters and wall thicknesses were measured from 2D images, ensuring a measurement perpendicular to the long axis of the ventricle. Pulsed wave Doppler at the apical position was used to record mitral inflow between the tips of the mitral leaflets and also, record tricuspid inflow between the tips of the tricuspid leaflets. Peak velocity of early (E) and atrial (A) diastolic filling and deceleration time (DT) of the E-wave were measured. E/A was calculated. LVEF was determined using M-mode (Table 1).

\section{2) Tissue Doppler Parameters}

Transthoracic echocardiography with tissue Doppler examination was performed before PCI ( 24 hours before the procedure) as well immediately after the 
Table 1. Conventional echocardiographic indices before PCI, as well as 1 day and 6 weeks after intervention.

\begin{tabular}{ccccc}
\hline Variables & Before PCI & 1 day after PCI & 6 weeks after PCI & P value \\
\hline LVEDD (cm) & $5.38 \pm 6.7$ & $5.33 \pm 5.9$ & $5.02 \pm 5.6$ & $<0.001$ \\
LVESD (cm) & $3.57 \pm 5.8$ & $3.53 \pm 5.5$ & $3.17 \pm 5.4$ & $<0.001$ \\
LVEF (\%) & $55.4 \pm 5.7$ & $55.6 \pm 5.2$ & $58.7 \pm 4.9$ & $<0.001$ \\
Transmitral E-wave (cm/s) & $56.24 \pm 2.5$ & $56.8 \pm 4.7$ & $59.94 \pm 2.2$ & 0.12 \\
Transmitral A-wave (cm/s) & $64.4 \pm 3.4$ & $62.7 \pm 4.4$ & $60.3 \pm 3.4$ & 0.19 \\
Transmitral E/A ratio & $0.87 \pm 0.26$ & $0.9 \pm 0.24$ & $0.92 \pm 0.25$ & 0.08 \\
Transmitral E-wave DT (ms) & $139.32 \pm 7$ & $135.26 \pm 9$ & $140.64 \pm 11$ & 0.12 \\
Transtricuspid E-wave (cm/s) & $41.44 \pm 2$ & $37.96 \pm 1.9$ & $44.04 \pm 1.9$ & 0.18 \\
Transtricuspid A-wave (cm/s) & $70.8 \pm 6.7$ & $67.66 \pm 8.2$ & $69.7 \pm 7$ & 0.38 \\
Transtricuspid E/A ratio & $0.59 \pm 0.23$ & $0.56 \pm 0.17$ & $0.63 \pm 0.20$ & 0.09 \\
Transtricuspid E-wave DT (ms) & $121 \pm 5$ & $127 \pm 9$ & $117 \pm 8$ & 0.17 \\
\hline
\end{tabular}

Data are expressed as mean \pm SEM. P values refer to repeated-measures ANOVA. (P-Value less than 0.05 were considered significant).

procedure 1 day after the procedure in all subjects. An additional tissue Doppler transthoracic echocardiographic examination was performed 6 weeks after PCI. All measurements were made by a single investigator. With the subject in the lateral supine position, Doppler recordings were obtained from the standard parasternal and apical views at the highest possible frame rate. The Doppler velocity range was accustomed between $-30 \mathrm{anf}+30 \mathrm{~cm} / \mathrm{s}$, with a sweep speed of 100 $\mathrm{mm} / \mathrm{s}$. The average of three successive cycles was used for the calculations of all echocardiographic Doppler parameters.

Peak longitudinal systolic (S'), early diastolic (E'), and late diastolic (A') velocities were measured (in centimeters per second) at the six mitral annular sites dividing the left ventricle into six segments of interest; the septal, lateral, anterior, inferior, posterior, and anteroseptal myocardial walls, as well as on the lateral angle of the tricuspid valve. Besides we also extracted data on mean differences in E/E' (the ratio of early diastolic trans-mitral and trans-tricuspid flow velocity and early diastolic tissue velocity) and E'/A' (the ratio of early and late diastolic tissue velocity), and calculation of myocardial performance index by TDI.

\section{The statistical analysis.}

Data were processed and analyzed using computer software SPSS (Statistical Package for Social Sciences) version 24 (SPSS Inc., Chicago, IL, USA). Continuous variables were expressed as means \pm SDs. Categorical variables expressed as frequency and corresponding percentages. To compare between the pre and post PCI groups, the continuous variables were analyzed using the repeated measure ANOVA test. The level of significance was set at 0.05 and $p<0.05$ was considered significant. To compare the pre and post PCI within the same group, 
the continuous variables were analyzed using the Friedman test.

\section{Results}

\section{Patient Characteristics}

Fifty consecutive patients with range, 45 - 75, 32 men with normal systolic left ventricular function undergoing elective PCI at our hospital were included in this study. Overall, 21 patients (42\%) had diabetes mellitus, 35 patients (70\%) had arterial hypertension, 5 patients (10\%) were obese, 13 patients $(26 \%)$ had dyslipidemia, 15 patients (30\%) had a family history of IHD and 10 patients (20\%) were smokers and 16 patients (32\%) were ex-smoker. 10 patients (20\%) had ischemic changes on their ECG as shown in Figure 1.

\section{Angiographic characteristics of the patients}

Single-vessel disease was present in 21 patients (in the left anterior descending [LAD] coronary artery in 18 , in the left circumflex coronary artery 1 , and in the right coronary artery [RCA] 2, 2 vessel disease in 25 patients, and 3-vessel disease in 4 patients as shown in Table $2 \&$ Figure 2. In total, 80 interventions were performed (LAD artery, $\mathrm{n}=42$; left circumflex artery, $\mathrm{n}=13$; RCA artery, $\mathrm{n}=$ 22) as shown in Tables 3-5 \& Figure 3. There were no procedural complications, nor were re-interventions required because of symptoms or objective evidence of ischemia.

\section{Conventional echocardiography}

Conventional echocardiographic data analysis revealed no statistically significant difference between pre- \& post-PCI (1 day after PCI and 6 weeks after PCI) as regarding trans-mitral (E, A, E/A ratio and $\mathrm{E} D T$ ) and trans-tricuspid (E, A, E/A ratio \& E DT) except as regarding LVEDD, LVESD and LVEF, there is statistically highly significant difference between pre- \& post-PCI 1 day after PCI and 6 weeks after PCI (P-value for each is $<0.001)$.

\section{Tissue Doppler Data}

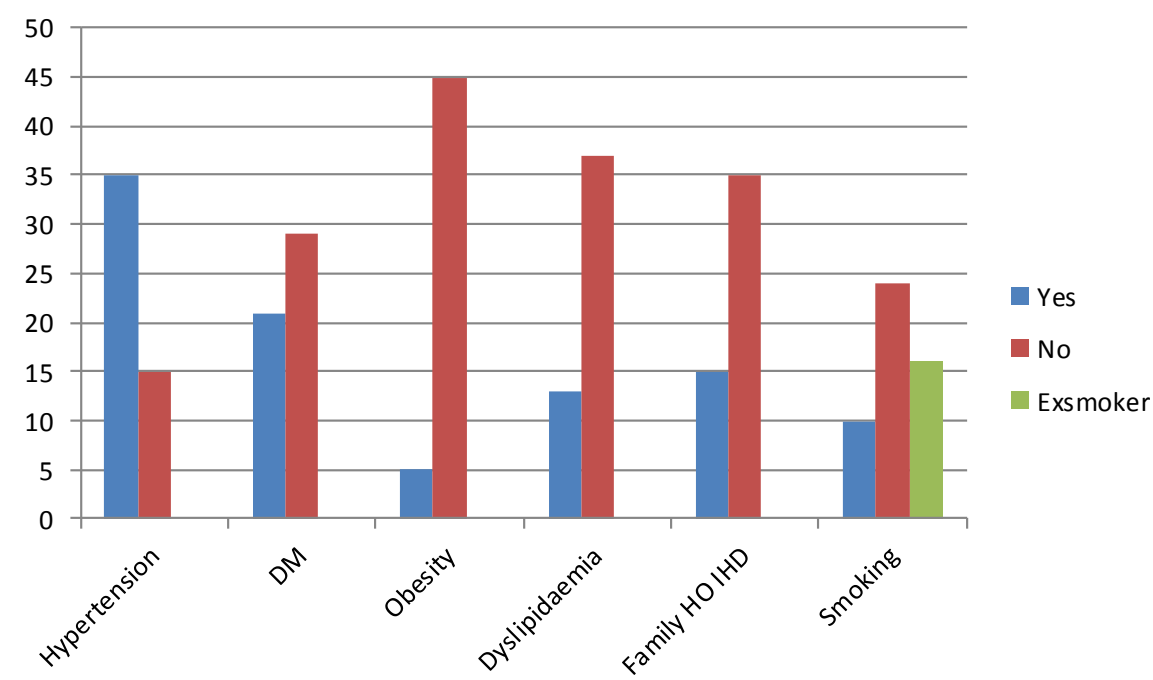

Figure 1. Risk factors differentiation \& patients characteristics. 


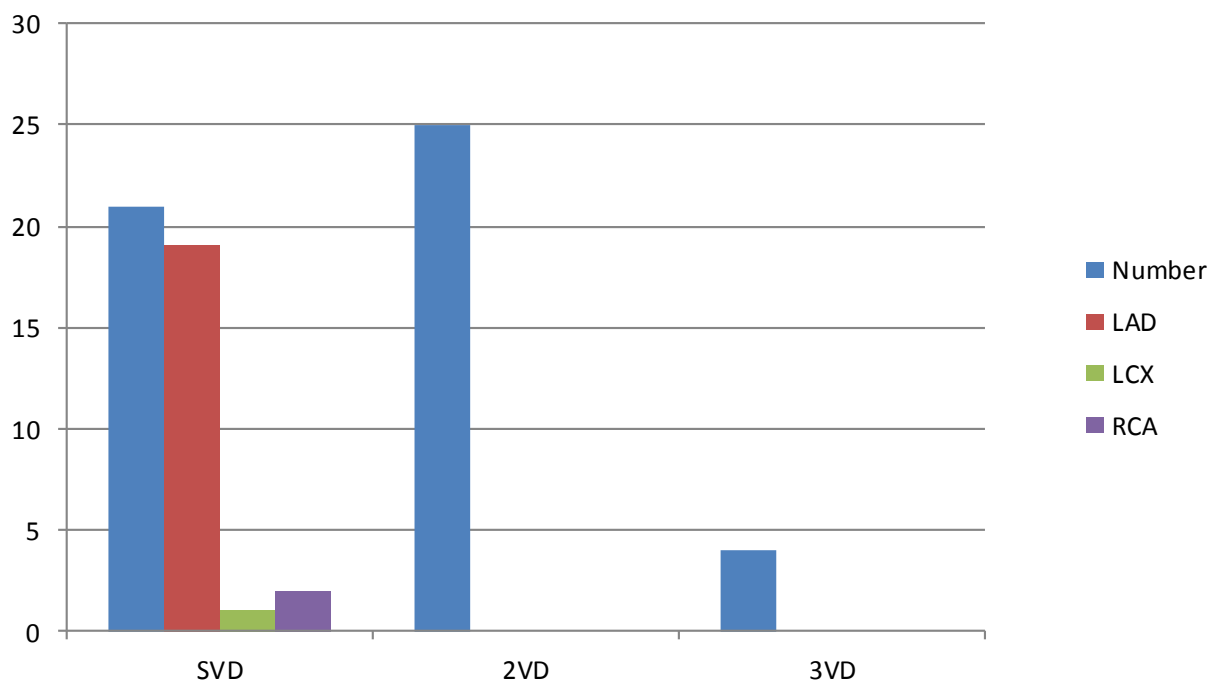

Figure 2. Angiographic results of the patients. Single-vessel disease was present in 21 patients (in the left anterior descending [LAD] coronary artery in 18, in the left circumflex coronary artery 1 , and in the right coronary artery [RCA] 2, 2 vessel disease in 25 patients, and 3-vessel disease in 4 patients.

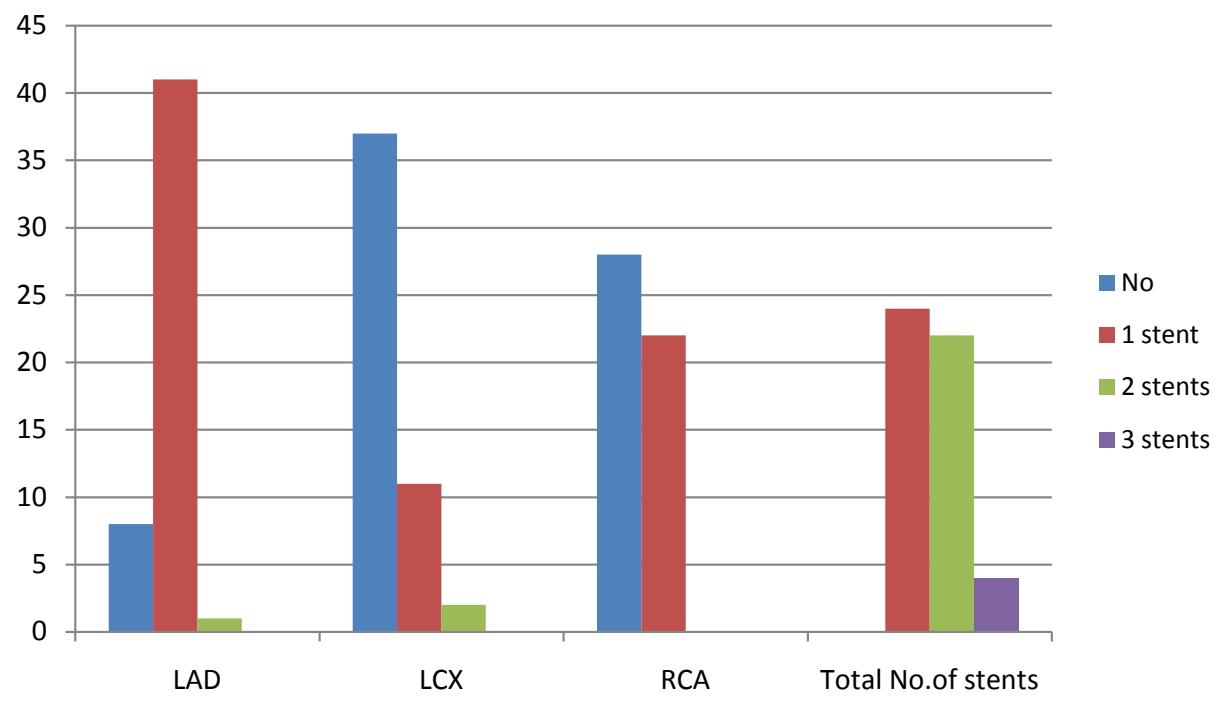

Figure 3. Angiographic data of the patients' PCI. Tables 3-5 \& figure shows total 80 interventions were performed (LAD artery, $\mathrm{n}=42$ ); left circumflex artery, $\mathrm{n}=13$; RCA artery, $\mathrm{n}=$ 22). There were no procedural complications, nor were reinterventions required because of symptoms or objective evidence of ischemia.

Table 2. Angiographic characteristics of patients.

\begin{tabular}{cc}
\hline Angiographic results: & Study population $(\mathrm{n}=50)$ \\
\hline Single vessel disease & $21(42 \%)$ \\
LAD & $18(36 \%)$ \\
LCX & $1(2 \%)$ \\
RCA & $2(4 \%)$ \\
2 vessels diseases & $25(50 \%)$ \\
3 vessels diseases & $4(8 \%)$ \\
\hline
\end{tabular}


Table 3. Stents in LAD number.

\begin{tabular}{ccc}
\hline & Frequency & Percent \% \\
\hline No stents & 8 & $16.0 \%$ \\
One stent & 41 & $82.0 \%$ \\
Two stents & 1 & $2.0 \%$ \\
Total & 50 & $100.0 \%$ \\
\hline
\end{tabular}

Table 4. Stents in LCX number.

\begin{tabular}{ccc}
\hline & Frequency & Percent \% \\
\hline No stents & 37 & $74.0 \%$ \\
One stent & 11 & $22.0 \%$ \\
Two stents & 2 & $4.0 \%$ \\
Total & 50 & $100.0 \%$ \\
\hline
\end{tabular}

Table 5. Stents in RCA number.

\begin{tabular}{ccc}
\hline & Frequency & Percent \% \\
\hline No stents & 28 & $54.0 \%$ \\
One stent & 22 & $46.0 \%$ \\
Total & 50 & $100.0 \%$ \\
\hline
\end{tabular}

Tissue Doppler Imaging (TDI) shows that systolic myocardial peak velocities improved significantly after PCI in the septal (ant.septal and post.septal), lateral, ant. and inferior walls (repeated-measures ANOVA P-value is $<0.05$ for each, especially is highly significant $<0.001$ for ant.septal S'). A non-significant trend toward an improvement in the posterior wall (repeated-measures ANOVA $P=$ $0.06)$ was found.

Tissue Doppler Imaging (TDI) shows that there was a significant improvement of early diastolic function E' after PCI at all parts of the mitral annulus (repeated-measures ANOVA P-value is significant $<0.05$ for post.septal and highly significant $<0.001$ for other). Also, there was a significant improvement of late diastolic function $\mathrm{A}^{\prime}$ after PCI at all parts of the mitral annulus (repeated-measures ANOVA P-value is significant $<0.05$ for each except at post. Wall A' ( $\mathrm{P}$ value is insignificant $=0.08$ ). Also, there was a significant improvement of E'DT \& E'/A' ratio after PCI (repeated-measures ANOVA P-value is significant $<0.05$ for each) but there was insignificant difference between pre- \& post PCI for E/E' (repeated-measures ANOVA P value $=0.154$ ).

LV cardiac time intervals by TDI M-mode revealed a significant reduction in IVRT, IVCT \& MPI after PCI (repeated-measures ANOVA P-value is significant $<0.05$ for each) but there was insignificant change in ET after PCI (repeated-measures ANOVA P value $=0.09$ )

LV Tissue Doppler Data in Patients with SVD (LAD) Pre- \& Post-PCI 
To estimate whether or not the improvement in myocardial left ventricular functions were associated or related to the revascularization procedure, we compared the myocardial velocities both before and after PCI in areas targeted by revascularization with areas outside the target areas. A sufficient number of patients to allow for a meaningful statistical analysis were present for interventions within the LAD artery $(n=18)$. Conventional echocardiographic data analysis revealed no statistically significant difference between pre- \& post-PCI(1 day and 6 weeks after PCI) as regarding trans-mitral (E, A, E/A ratio \& E DT) except as regarding LVEDD, LVESD and LVEF (by Friedman Test, P-value < 0.05 for each).

In this group, there was a significant improvement in systolic function $S^{\prime}$ wave velocities at ant.septal and ant. Walls (by Friedman Test, P-value $<0.05$ for each). There was a significant improvement in early diastolic function E' wave velocities at ant., ant.septal, post.septal \& post. Walls (by Friedman Test, P-value $<0.05$ for each \& especially P-value is highly significant $<0.001$ for E' at ant. walls). But there was no significant improvement in late diastolic function $\mathrm{A}^{\prime}$ wave velocities at all the angles of the mitral annulus but there was a slight and insignificant improvement toward A' at the ant. Wall (by Friedman Test, P-value $=0.06$ ). As regarding, other diastolic parameters and cardiac time intervals in these patients which were performed at the septal angle of the mitral annulus showing that there was a significant improvement in E'DT, E'/A', E/E', IVRT and IVCT (by Friedman Test, P-value $<0.05$ for each) but there was an insignificant change in ET and MPI (by Friedman Test, P-value $>0.05$ for each).

\section{$R V$ Tissue Doppler Data}

The RV shows a significant improvement in tissue Doppler measures of systolic function S' after PCI compared with baseline values (repeated measures ANOVA P-value is highly significant $<0.001$ ). Also, there was a significant improvement in tissue Doppler measures of early, late diastolic function and E'/A' after PCI compared with baseline values (repeated measures ANOVA P-value is highly significant $<0.001$ for E' \& P-value is $<0.05$ for A' \& E'/A') but there was an insignificant change in tissue Doppler measures of E'DT \& E/E' after PCI (repeated measures ANOVA P-value is $>0.05$ for each).

RV cardiac time intervals by TDI M-mode revealed a significant reduction in IVRT, IVCT \& MPI after PCI (repeated-measures ANOVA P-value is significant $<0.05$ for each) but there was insignificant change in ET after PCI (repeated-measures ANOVA P value $=0.554)$.

\section{$R V$ Tissue Doppler Data in Patients with or without RCA Intervention}

TDI shows that there was a significant improvement in tissue Doppler measures of systolic and diastolic function, both in patients undergoing intervention within the RCA territory $(n=22)$ and without RCA intervention ( $\mathrm{n}=$ 28). There was a significant improvement in tissue Doppler measures of systolic S' in patients with \& without RCA intervention between pre- \& post-PCI (repeated-measures ANOVA P-value is $<0.05$ for each). Also, there was a highly significant improvement in tissue Doppler measures of early diastolic function 
E' in both groups between pre- \& post-PCI (repeated-measures ANOVA P-value is $<0.001$ for each). There was a significant improvement in tissue Doppler measures of late diastolic function $\mathrm{A}^{\prime}$ and $\mathrm{E} / \mathrm{E}^{\prime}$ ratio in patients with RCA intervention between pre- \& post-PCI (repeated-measures ANOVA P-value is $<0.05$ for it) but There was no significant improvement in tissue Doppler measures of late diastolic function $\mathrm{A}^{\prime}$ and $\mathrm{E} / \mathrm{E}^{\prime}$ ratio in patients without RCA intervention between pre- \& post-PCI (repeated-measures ANOVA P-value is $>0.05$ for it).

There was no significant change in tissue Doppler measures of E'DT in patients with or without RCA intervention between pre- \& post-PCI (repeated-measures ANOVA P-value is $>0.05$ for each). There was a significant change in tissue Doppler measures of E'/A' ratio in patients without \& with RCA intervention between pre- \& post-PCI (repeated-measures ANOVA P-value is $<0.05 \&$ is highly significant $<0.001$, respectively).

As regarding, RV cardiac time intervals by TDI M-mode before \& after PCI revealed a difference between patients with or without RCA intervention. In patients with RCA intervention, there was a significant change in IVRT, IVCT \& MPI (repeated-measures ANOVA P-value is $<0.05$ for each) but there was no significant change in ET (repeated-measures ANOVA P-value is $>0.05$ for it). In patients without RCA intervention, there was a significant change in ET (repeated-measures ANOVA P-value is $<0.05$ for it) but there was no significant change in IVRT, IVCT \& MPI (repeated-measures ANOVA P-value is $>0.05$ for each).

\section{Discussion}

The purpose of this study was to investigate the impact of the percutaneous coronary intervention (PCI) on myocardial function in patients with chronic stable angina included a total of 50 patients, assessed by tissue Doppler echocardiography, and its target also was to find out if after PCI longitudinal and circumferential myocardial impairment improved or not. The concept of hibernating myocardium was introduced a decade ago by Braunwald and Rutherford [7] and Rahimtoola [8]. The term describes persistent myocardial dysfunction at rest because of under perfusion, with subsequent improvement upon revascularization [7] [8]. It has been hypothesized that the gold-standard approval for myocardial hibernation is the enhancement in segmental myocardial performance after revascularization [9]. Although cases that included in the Henein et al. [10] study with stable angina had normal systolic function, they actually had longitudinal dysfunction, chiefly in the subendocardial layer. Thus, improved coronary blood and thereby oxygen supply may lead to improved long-axis function. Longitudinally orientated myocardial fibers are mainly positioned subendocardially; which is the most vulnerable area to ischemia. That is why longitudinal measures are finely sensitive indices of ischemia [10].

\section{Improvement in Left Ventricular Myocardial Function after PCI}

In our study, there was an improvement in the left ventricular myocardial 
function after PCI. Both diastolic and systolic function improved within few hours after successful angioplasty, and this effect persists for over six weeks after intervention. This proposed that, even with normal baseline cardiac function, reduced regional contractile function may exist in patients with chronic stable angina, with subsequent improvement of the cardiac functions after successful revascularization. Previous studies proved similar early impairment of the longitudinal systolic function in patients with preserved ejection fraction [11] [12].

The present study, therefore, was designed to estimate the effects of PCI on diastolic dysfunction in CAD patients early and late after PCI. Previous studies have suggested that balloon angioplasty and stenting augment the statistical parameters related to left ventricular diastolic function even 48 hours after elective PCI [13].

Our study is consistent with Diller et al. [14] study; who examined 24 patients with chronic stable angina and preserved systolic left ventricular function who underwent PCI; patients had PW-TDI and conventional echocardiography before PCI and after 1 day and 6 weeks after the procedure. They found that all TDI measurements of early diastolic function improved significantly after PCI with the most noticeable improvement was in the septal area; similar to our study. Also, they stated that systolic myocardial peak velocities improved markedly after PCI in wach of the septal, lateral, and inferior walls but this was less evident regarding the improvement in the posterior wall. In our study, however, systolic myocardial peak velocities $S$ ' at all the angles of the mitral annulus improved significantly after PCI. Also, they found that all TDI measurements of late diastolic function didn't improve significantly after PCI except for septal A', which is different from our study as we found that late diastolic myocardial peak velocities improved significantly after PCI at all the parts of the mitral annulus except for post. A'.

Also, Diller et al. study didn't include those parameters (E'/A', E/E', E' DT \& cardiac time intervals) in their study. Diller et al. were agreed with our study showing that trans-mitral and trans-tricuspid flow Doppler parameters failed to reflect any significant change 1 day after PCI and 6 weeks after PCI. He didn't agree with our study that showed that $\mathrm{EF}$ failed to reflect any improvement 1 day or 6 weeks after PCI [14].

Tümüklü et al. [15] studied the systolic and diastolic functions for 21 patients with stable CAD and LVEF $>40 \%$ before and 48 hours after successful elective PCI. They were agreed with our study showing that trans-mitral flow Doppler parameters failed to reflect any significant change after PCI. They didn't agree with our study that showed that systolic indices and early and late diastolic velocities of the mitral annulus in TDI failed to reflect any improvement after PCI. They didn't follow the patients again for a prolonged time as we have done in our study and that cause may be responsible for the minor correlation differences between these two studies.

Hossain et al. [16] study was done on 40 consecutive patients who agreed with our study showed that there were significant differences in that conventional 
echocardiographic measure (LVEDD, LVESD and LVEF) between baseline and 6 weeks following PCI. He didn't perform trans-mitral or trans-tricuspid flow Doppler parameters as in our study at all that were insignificant. They were agreed with our study showing that there was a significant improvement in E'-wave, A'-wave and E'/A' ratio at the lateral mitral annulus 6 weeks after PCI. The deceleration time (E' DT m) also exhibited significant change 6 weeks after PCI but there are differences between their study and our study in that they measured these parameters only on the lateral mitral annulus \& they didn't include these parameters (E/E' \& IVCT, MPI \& ET) as we have done. They did not agree with our study showing that there was an insignificant improvement in $S^{\prime}$ wave \& IVRT at the lateral mitral annulus 6 weeks after PCI.

Tanaka et al. [17] study done on 27 consecutive patients who wasn't agree with our study, showed that there were no significant differences in that conventional echocardiographic measures (LVEDD, LVESD and LVEF) 24 hours before and $22 \pm 8$ hours thereafter PCI, in contrast with our study, we have found significant results within six weeks, This temporal discrepancy in echocardiographic examination time may be responsible for the minor correlation differences between these two studies. Also, the study found slight but not significant increases in mitral flow E and E/A ratio with PCI in all 27 patients which that was agreed with our study.

As we mentioned before that unlike conventional visual assessment of regional wall motion abnormalities, requiring subjective interpretation, tissue Doppler velocities can be quantified objectively. Because of the limited utility of transmitral flow profile in the evaluation of the LV diastolic function, recently, assessment of myocardial velocities by Doppler tissue imaging is gaining importance.

Most of these above studies proved that the development of ischemia is associated with a progressive reduction in systolic contraction and the earliest echocardiographic abnormalities observed in chronic stable angina is the relaxation disorder characterized by the reduction in the E/A ratio becoming $<1$, the decrease in $\mathrm{E}$ wave, the increase in A wave and increase in E DT and IVRT. Studies suggest that such a pattern is primarily the result of impairment of the energy-requiring process of ventricular relaxation and experimental and clinical studies have revealed improvements of diastolic function after percutaneous transluminal coronary angioplasty (PTCA) in both early and late post-PCTA follow up measurements [18].

These studies suggested that the improvement in left ventricular early diastolic function after PCI might be associated with the degree of improvement in impaired regional myocardial relaxation. Increased compliance after PTCA may reflect a decrease in reversible myocardial ischemia. Furthermore, our results showed that improvement in myocardial relaxation in CAD caused by PCI and that regional myocardial relaxation is associated with the improvement in dynamics of global LV early diastolic filling.

Most of the studies were in agreement with our study that PW-TDI could diagnose resting regional ischemia in a patient with stable CAD despite normal 
wall motion and $\mathrm{EF}$ and also can detect improvement of regional function after elective PCI to significant stable CAD.

\section{Effect of LAD stenting on $L t V$. function}

Bayata et al. [19] studied on thirty patients with isolated severe left anterior descending coronary artery stenosis and normal systolic function. And also, $R i-$ cou et al. [20] who agreed with our study showing that trans-mitral flow Doppler parameters failed to reflect any significant change 1 day and 3 months after PCI, respectively.

In contrast, Masuyama et al. [21] using pulsed Doppler echocardiography in 50 patients with stable exertional angina pectoris. Peak rapid filling velocity E and the ratio of peak atrial filling to peak rapid filling velocities E/A were measured from the transmitral flow velocity pattern before and 2 and 9 days after coronary angioplasty. They didn't agree with our study that reported an improvement of these parameters within two days after angioplasty and continued to improve up to 9 days.

In these studies, they didn't agree with our study that showed that EF failed to reflect any improvement after PCI. Also, they didn't perform PW-TDI as in our study we have done.

\section{Improvement in Right Ventricular Myocardial Function after PCI}

Our study is consistent with Diller et al. [14] and Tümüklü et al. [15] who showed that all tissue Doppler measurements of systolic and early and late diastolic function at the lateral angle of the tricuspid annulus improved significantly after PCI.

\section{$R V$ Tissue Doppler Data in Patients with or without RCA Intervention}

Analysis of the effect of target vessel revascularization on right ventricular longitudinal function in the current study was hampered by the fact that only 22 patients underwent revascularization in the RCA territory. However, our study shows that there was a significant improvement in tissue Doppler measures of systolic and diastolic function, both in patients undergoing intervention within the RCA territory $(\mathrm{n}=22)$ and to without RCA intervention $(\mathrm{n}=28)$. In our study, there was a significant improvement in tissue Doppler measures of systolic and diastolic function in patients with and without RCA intervention between pre- \& post-PCI tended to be more pronounced in patients with RCA intervention.

Our study is consistent with Diller et al. [14] study showing that improvement in tissue Doppler measures of systolic and diastolic function was found, both in patients undergoing intervention within the RCA territory $(n=7)$ and to a lesser extent in patients without RCA intervention $(\mathrm{n}=17)$ Also, Diller et al. [14] study didn't include that parameters (E'/A', E/E', E' DT and cardiac time intervals) in their study.

These results may be due to 2 independent mechanisms which may be responsible for improved right ventricular function after PCI. It appears likely that besides the direct effect of augmented blood supply to the right ventricle as a result of successful PCI, right ventricular function improves as a consequence of 
augmented left ventricular function (ventriculo-ventricular interaction), affecting biventricular geometry or improved left ventricular diastolic function and leading to reduced right ventricular afterload.

\section{Conclusions and Recommendations}

Tissue Doppler echocardiography is a noninvasive and widely available diagnostic technique that allows the sensitive detection of myocardial dysfunction. PW-TDI could diagnose resting regional ischemia in a patient with stable CAD despite normal wall motion and $\mathrm{EF}$ and also can detect improvement of regional function after elective PCI to significant stable CAD. PCI for a significant coronary lesion has a beneficial effect on LV functions, and this work emphasizes its value in the improvement of regional and global LV functions \& myocardial contractility that can be predicted by conventional echo and TDI.

This small scale study may play a significant role in making more conclusive large scale studies with long-term follow-up in multiple center study.

\section{Limitations of the Study}

The limited number of patients, short-term follow-up, and the fact that this was a single-center study could limit the strength of the results. Also, we used only tissue Doppler velocity imaging at the atrioventricular valve annulus to assess longitudinal ventricular function. Tissue Doppler imaging is relatively angle-dependent which means that if the angle of interrogation exceeds 20 degrees, the velocity may be underestimated. In fact, the Doppler technique depends on parallel alignment of the Doppler beam to the moving objects and angulations can disturb the results. However, this can be lessened by choosing the region of interest within $20^{\circ}$ of the axis of the Doppler interrogation. Future larger studies are recommended to prove or deny our results and also it is advised that future studies should compare changes in myocardial velocities in areas targeted by the intervention to those in areas outside the revascularized myocardium.

\section{Conflicts of Interest}

The authors declare no conflicts of interest regarding the publication of this paper.

\section{References}

[1] Smith Jr., S.C., Feldman, T.E., Hirshfeld Jr., J.W., Jacobs, A.K., Kern, M.J., King 3rd, S.B., et al. (2006) ACC/AHA/SCAI 2005 Guideline Update for Percutaneous Coronary Intervention-Summary Article: A Report of the American College of Cardiology/American Heart Association Task Force on Practice Guidelines (ACC/AHA/SCAI Writing Committee to Update the 2001 Guidelines for Percutaneous Coronary Intervention). Journal of the American College of Cardiology, 47, 216-235.

[2] Veyrat, C., Larrazet, F., Cohen, L., Laborde, F. and Pellerin, D. (2005) Detection of Prominent Left Anterior Descending Coronary Artery Stenosis for Patients with 
Stable Angina Using Doppler Tissue Echocardiography. Journal of the American Society of Echocardiography, 18, 821-829.

https://doi.org/10.1016/j.echo.2004.12.025

[3] Wang, J., Abraham, T.P., Korinek, J., Urheim, S., McMahon, E.M. and Belohlavek, M. (2005) Delayed Onset of Subendocardial Diastolic Thinning at Rest Identifies Hypoperfused Myocardium. Circulation, 111, 2943-2950. https://doi.org/10.1161/CIRCULATIONAHA.104.482984

[4] Henein, M.Y., Priestley, K., Davarashvili, T., Buller, N. and Gibson, D.G. (1993) Early Changes in Left Ventricular Subendocardial Function after Successful Coronary Angioplasty. British Heart Journal, 69, 501-506. https://doi.org/10.1136/hrt.69.6.501

[5] Schiller, N.B., Shah, P.M., Crawford, M., DeMaria, A., Devereux, R., Feigenbaum, H., et al. (1989) Recommendations for Quantitation of the Left Ventricle by Two-Dimensional Echocardiography. American Society of Echocardiography Committee on Standards, Subcommittee on Quantitation of Two-Dimensional Echocardiograms. Journal of the American Society of Echocardiography, 2, 358-367. https://doi.org/10.1016/S0894-7317(89)80014-8

[6] Edvardsen, T., Urheim, S., Skulstad, H., Steine, K., Ihlen, H. and Smiseth, O.A. (2002) Quantification of Left Ventricular Systolic Function by Tissue Doppler Echocardiography: Added Value of Measuring Pre- and Postejection Velocities in Ischemic myocardium. Circulation, 105, 2071-2077. https://doi.org/10.1161/01.CIR.0000014614.63980.BA

[7] Braunwald, E. and Rutherford, J.D. (1986) Reversible Ischemic Left Ventricular Dysfunction: Evidence for the "Hibernating Myocardium". Journal of the American College of Cardiology, 8, 1467-1470. https://doi.org/10.1016/S0735-1097(86)80325-4

[8] Rahimtoola, S.H. (1994) Chronic Myocardial Hibernation. Circulation, 89, 1907-1908. https://doi.org/10.1161/01.CIR.89.4.1907

[9] Ferrari, R., Ferrari, F., Benigno, M., Pepi, P. and Visioli, O. (1998) Hibernating Myocardium: Its Pathophysiology and Clinical Role. Molecular and Cellular Biochemistry, 186, 195-199. https://doi.org/10.1023/A:1006872827169

[10] Henein, M.Y. and Gibson, D.G. (1999) Normal Long Axis Function. Heart, 81, 111-113. https://doi.org/10.1136/hrt.81.2.111

[11] Bolognesi, R., Tsialtas, D., Barilli, A.L., Manca, C., Zeppellini, R., Javernaro, A., et al. (2001) Detection of Early Abnormalities of Left Ventricular Function by Hemodynamic, Echo-Tissue Doppler Imaging, and Mitral Doppler Flow Techniques in $\mathrm{Pa}$ tients with Coronary Artery Disease and Normal Ejection Fraction. Journal of the American Society of Echocardiography, 14, 764-772. https://doi.org/10.1067/mje.2001.113234

[12] Hoffmann, S., Mogelvang, R., Olsen, N.T., Sogaard, P., Fritz-Hansen, T., Bech, J., et al. (2010) Tissue Doppler Echocardiography Reveals Distinct Patterns of Impaired Myocardial Velocities in Different Degrees of Coronary Artery Disease. European Journal of Echocardiography, 11, 544-549.

https://doi.org/10.1093/ejechocard/jeq015

[13] Schannwell, C.M., Schneppenheim, M., Plehn, G. and Strauer, B.E. (2003) Parameters of Left Ventricular Diastolic Function 48 Hours after Coronary Angioplasty and Stent Implantation. Journal of Invasive Cardiology, 15, 326-333.

[14] Diller, G.P., Wasan, B.S., Thom, S.A., Foale, R.A., Hughes, A.D., Francis, D.P., et al. (2009) Evidence of Improved Regional Myocardial Function in Patients with 
Chronic Stable Angina and Apparent Normal Ventricular Function-A Tissue Doppler Study before and after Percutaneous Coronary Intervention. Journal of the American Society of Echocardiography, 22, 177-182. https://doi.org/10.1016/j.echo.2008.10.018

[15] Tümüklü, M., Kayikçioğlu, M., Aliyev, E., Cinar, C.S. and Soydan, I. (2003) [Evaluation of Early Alterations in Transmitral Diastolic Flow and Tissue Doppler Findings of the Basal Segments of Both Ventricles in Early Period after Coronary Angioplasty]. Anadolu Kardiyoloji Dergisi, 3, 16-23.

[16] Hossain, M.M., Rahman, A., Siddique, M.A., Banerjee, S., Ahmed, C., Hoque, H., et al. (2013) A study of Changes in Various Echocardiographic Parameters in Patients with Chronic Stable angina undergoing Percutaneous Coronary Intervention (PCI). University Heart Journal, 9, 99-106. https://doi.org/10.3329/uhj.v9i2.23431

[17] Tanaka, H., Kawai, H., Tatsumi, K., Kataoka, T., Onishi, T., Nose, T., Mizoguchi, T. and Yokoyama, M. (2006) Improved Regional Myocardial Diastolic Function Assessed by Strain Rate Imaging in Patients with Coronary Artery Disease Undergoing Percutaneous Coronary Intervention. Journal of the American Society of Echocardiography, 19, 756-762. https://doi.org/10.1016/j.echo.2006.01.008

[18] Devereux, R.B. (1989) Left Ventricular Diastolic Dysfunction: Early Diastolic Relaxation and Late Diastolic Compliance. Journal of the American College of Cardiology, 13, 337-339. https://doi.org/10.1016/0735-1097(89)90508-1

[19] Bayata, S., Susam, I., Pinar, A., Dinckal, M.H., Postaci, N. and Yesil, M. (2000) New Doppler Echocardiographic Applications for the Evaluation of Early Alterations in Left Ventricular Diastolic Function after Coronary Angioplasty. European Journal of Echocardiography, 1, 105-108. https://doi.org/10.1053/euje.2000.0021

[20] Ricou, F., Lerch, R., Meier, B. and Rutishauser, W. (1992) Abnormal Left Ventricular Filling Pattern in Patients with Single Vessel Coronary Artery Disease: Effect of Angioplasty. Cardiology, 80, 230-236. https://doi.org/10.1159/000175007

[21] Masuyama, T., Kodama, K., Nakatani, S., Nanto, S., Kitabatake, A. and Kamada, T. (1988) Effects of Changes in Coronary Stenosis on Left Ventricular Diastolic Filling assessed with Pulsed Doppler Echocardiography. Journal of the American College of Cardiology, 11, 744-751. https://doi.org/10.1016/0735-1097(88)90206-9 